\title{
Evaluating the effects of dexmedetomidine compared to propofol as adjunctive therapy in patients with alcohol withdrawal
}

This article was published in the following Dove Press journal:

Clinical Pharmacology: Advances and Applications

31 October 2014

Number of times this article has been viewed

\section{Riley J Lizotte' \\ John A Kappes ${ }^{2}$ \\ Billie J Bartel ${ }^{2}$ \\ Katie M Hayes' \\ Veronica L Lesselyoung'}

'Pharmacy Department, Rapid City Regional Hospital, Rapid City, SD, USA; ${ }^{2}$ Pharmacy Practice, South

Dakota State University,

Brookings, SD, USA
Correspondence: Riley J Lizotte The Aroostook Medical Center, Pharmacy Department, 140 Academy St, Presque Isle, ME 04769, USA Email rlizotte@emhs.org
Background: In severe alcohol withdrawal (AW), benzodiazepines may be inadequate to control symptoms. In many situations, benzodiazepine dosing escalates despite no additional efficacy and introduces potential toxicities. Severe cases of AW may require additional agents to control symptoms. Case reports and studies have shown benefits with dexmedetomidine and propofol in severe AW, but these agents have not been compared with one another. This study compares the effects of dexmedetomidine and propofol on benzodiazepine and haloperidol utilization in patients with AW.

Methods: A retrospective chart review was completed on 41 patients with AW who received adjunctive dexmedetomidine or propofol. The primary objective was to compare benzodiazepine and haloperidol utilization before and after initiation of dexmedetomidine or propofol. Secondary measures included AW and sedation scoring, analgesic use, intensive care unit length of stay, rates of intubation, and adverse events.

Results: Among the dexmedetomidine and propofol groups, significant reductions in benzodiazepine ( $P \leq 0.0001$ and $P=0.043$, respectively) and haloperidol $(P \leq 0.0001$ and $P=0.026$, respectively) requirements were observed. These reductions were comparable between groups ( $P=0.933$ and $P=0.465$, respectively). A trend toward decreased intensive care unit length of stay in the dexmedetomidine group ( 123.6 hours vs 156.5 hours; $P=0.125$ ) was seen. Rates of intubation ( $14.7 \%$ vs $100 \%$ ) and time of intubation (19.9 hours vs 97.6 hours; $P=0.002$ ) were less in the dexmedetomidine group. Incidence of hypotension was $17.6 \%$ in the dexmedetomidine group vs $28.5 \%$ in the propofol group. Incidence of bradycardia was $17.6 \%$ in the dexmedetomidine group vs $0 \%$ in the propofol group. No differences were observed in other secondary outcomes.

Conclusion: In patients with severe AW who require sedation, both dexmedetomidine and propofol have unique and advantageous properties. Both agents appear to have equivalent efficacy in reducing AW-related symptoms and benzodiazepine and haloperidol requirements. These results should be validated in a larger, prospective trial.

Keywords: dexmedetomidine, propofol, benzodiazepines, alcohol, withdrawal

\section{Introduction}

Alcohol dependence is a common concern during hospitalization, affecting an estimated $15 \%-20 \%$ of patients. ${ }^{1}$ Chronic alcohol use leads to modulation of the central nervous system. $N$-Methyl-D-aspartate (NMDA) receptors are upregulated, while gamma-aminobutyric acid (GABA) receptors become downregulated. ${ }^{2}$ Temporary absence of alcohol in this setting results in hyperactivity due to enhanced NMDA function, reduced GABA stimulation, and dysregulation of the dopaminergic system, ${ }^{2}$ all of which contribute to various alcohol withdrawal (AW) symptoms. 
AW manifests from alcohol dependence, and symptomatology varies greatly among patients. The most frequent symptoms result from noradrenergic hyperactivity and include tremulousness, sweating, hypertension, tachycardia, and agitation. ${ }^{3,4}$ These symptoms typically do not require medical intervention, although severe cases of AW may necessitate medical care. In severe cases of AW, patients may suffer from neuronal excitation, such as epileptiform seizures, delirium tremens, and the possibility of death due to severe pulmonary and cardiovascular toxicities. ${ }^{3,4}$ Symptom presentation begins after 6-24 hours of abstinence with a typical duration of 48-72 hours, although some patients may experience longer withdrawal periods. ${ }^{1-4}$

The first-line pharmacologic agents for AW management are benzodiazepines due to their pharmacological action of enhancing GABA-mediated inhibition. ${ }^{1,2}$ They effectively alleviate symptoms while acting to treat and prevent AW seizures due to influx of chloride ions. The influx of anions causes hyperpolarization and creates an inhibitory effect on action potential firing. ${ }^{5,6}$ Despite these benefits, patients with severe cases of AW can be refractory to standard symptomtriggered benzodiazepine dosing. ${ }^{7}$ In such cases, dosing continues to increase as the patient remains symptomatic, a consequence of misunderstanding and non-cognizance of AW severity and pathology, in addition to protocol standardization. Such circumstances produce benzodiazepine overuse, ${ }^{8-10}$ potentially causing excessive sedation, insomnia, delirium, and respiratory depression. ${ }^{11,12}$

Propofol is a sedative agent that enhances GABA activity, similar to benzodiazepines. However, propofol binds at a different receptor site compared to benzodiazepines. ${ }^{6,13}$ In comparison to benzodiazepines for sedation, propofol may decrease time to extubation, ${ }^{14,15}$ and has displayed antiepileptic properties in refractory seizures, ${ }^{16-20}$ although in patients with AW has not been shown to shorten duration of AW or hospital length of stay. ${ }^{21}$

Dexmedetomidine is a centrally acting $\alpha-2$ adrenergic agonist used for sedation in both intubated and nonintubated patients. ${ }^{22,23}$ Dexmedetomidine has analgesic properties, does not cause respiratory depression, ${ }^{23-26}$ and has been shown to decrease duration of mechanical ventilation compared to other sedative agents..$^{27-29}$

Recent studies and case reports using propofol, ${ }^{18-21,30}$ and more recently dexmedetomidine, have shown reductions in benzodiazepine requirements and symptom control in patients with AW refractory to benzodiazepine therapy alone. ${ }^{31-37}$ To date, these agents have not been compared against each other. The goal of this study is to compare the effects of dexmedetomidine and propofol on benzodiazepine and haloperidol requirements in patients with AW.

\section{Methods \\ Patient selection}

Data were obtained via electronic medical record, identifying 124 patients who had orders for the AW order set and concurrent use of either dexmedetomidine or propofol for sedation from November 2010 to October 2013. Patients were included if they had a diagnosis of AW, received either sedative agent, and were actively receiving the institution's AW order set. Patients were excluded if they had AW orders discontinued before start of a sedative agent, age less than 18 years, scheduled benzodiazepine or antipsychotic continued from home, concomitant administration of a continuous infusion benzodiazepine, contraindication to using benzodiazepines or antipsychotics, or immediately received either sedative agent within 4 hours of hospital admission. Patients who received both dexmedetomidine and propofol were also excluded from the analysis (Figure 1).

\section{Study protocol}

This retrospective study was Institutional Review Board approved, and informed consent was waived. Data were collected from patients admitted to the medical, cardiac, surgical, or transitional intensive care units (ICUs) (27 beds total). The AW protocol contains intravenous fluids, thiamine, vitamins, haloperidol, and lorazepam. Lorazepam dosing correlates to the Alcohol Withdrawal Assessment Scale (AWAS), which evaluates tremor, tachycardia, blood pressure, diaphoresis, fever, nausea and vomiting, agitation, confusion or disorientation, sleeplessness, and hallucinations, each on a scale of $0-3$ (except sleeplessness, which has a scale of 0-2) for a maximum score of 29. Intravenous lorazepam is preferred in patients with an AWAS score more than 11 . Scoring is performed every 30 minutes by nursing, and lorazepam dosing is given based on the current score. Haloperidol may be used every 30 minutes as needed for "severe agitation." Dexmedetomidine and propofol are not listed on the AW protocol, and initiation of therapy is determined by provider judgment. Standard hospital dosing and titration protocols were used for dexmedetomidine and propofol. These protocols do not utilize bolus dosing.

\section{Data collection}

Eligible patients were analyzed based on the choice of sedative agent (ie, dexmedetomidine or propofol). Patient data were collected 24 hours before to 24 hours after initiation 


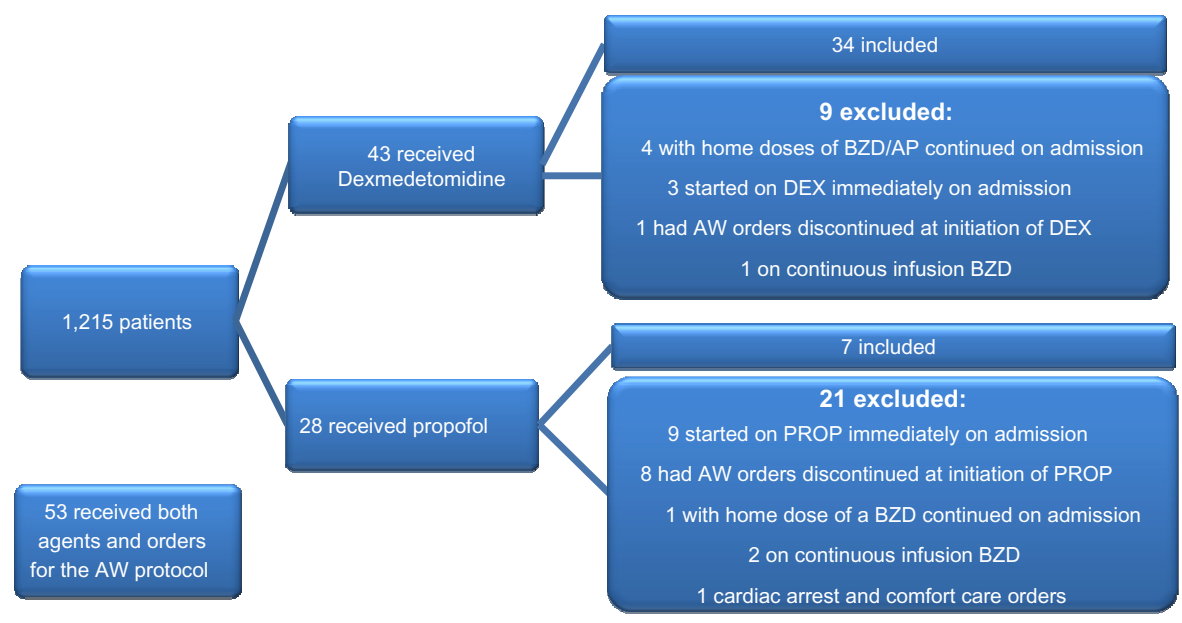

Figure I Patient population.

Abbreviations: BZD, benzodiazepine; AP, antipsychotic; AW, alcohol withdrawal; DEX, dexmedetomidine; PROP, propofol.

of the sedative agent (Figure 2). If a patient was started on sedation within the first 24 hours of admission, data were collected for the same time period following initiation of the sedative. The primary objective was to compare benzodiazepine and haloperidol utilization before and after initiation of dexmedetomidine or propofol. Benzodiazepine use is expressed in lorazepam equivalence (Table 1). ${ }^{38}$ Secondary objectives included ICU length of stay, rates and time of intubation, analgesic usage, AWAS score, Richmond Agitation Sedation Scale (RASS), Confusion Assessment Method for the ICU (CAM-ICU), and incidence of bradycardia and hypotension. Bradycardia was defined as a heart rate less than $60 \mathrm{bpm}$ (beats per minute), and hypotension as a systolic blood pressure less than $90 \mathrm{mmHg}$.

\section{Statistical analysis}

Statistical analyses were performed using SPSS Statistics 21 (IBM Corporation, Armonk, NY, USA). Two-tailed Wilcoxon signed-rank tests were used to assess significances between 24-hour before and 24-hour after initiation of sedation with benzodiazepine and haloperidol. Two-tailed Mann-Whitney $U$ tests assessed whether benzodiazepine use, haloperidol use, rates and time of intubation, and ICU length of stay differed between the dexmedetomidine and propofol groups. A $P$-value of less than 0.05 was considered statistically significant.

\section{Results}

Of the 124 patients who received orders for the AW protocol and the use of a sedative agent, 41 were included in the analysis. Fifty-three of the patients used dexmedetomidine and propofol concurrently and were excluded.
Twelve received dexmedetomidine or propofol immediately on admission, nine had AW protocol discontinued upon initiation of sedation, five had a benzodiazepine or antipsychotic continued from home, three received a continuous infusion of a benzodiazepine, and one was placed on comfort care within 6 hours of AW protocol initiation (Figure 1).

\section{Benzodiazepine and haloperidol dosing}

Nine patients $(26.4 \%)$ received a benzodiazepine other than lorazepam in the dexmedetomidine group, compared to three patients $(42.9 \%)$ in the propofol group, and were converted to lorazepam equivalency. Mean benzodiazepine and haloperidol use during the 24-hour period before initiation of sedation in the dexmedetomidine group was $20.9 \mathrm{mg}$ and $8.5 \mathrm{mg}$, respectively. This was similar to the propofol group, where mean benzodiazepine and haloperidol use was $17.4 \mathrm{mg}$ and $8.7 \mathrm{mg}$, respectively, prior to initiation of sedation (Figure 3). In the 24-hour period following initiation of sedation, benzodiazepine and haloperidol requirements were decreased to $4.4 \mathrm{mg}$ and $0.9 \mathrm{mg}$ in the dexmedetomidine group ( $P \leq 0.0001$ and $P \leq 0.0001)$, and $3.9 \mathrm{mg}$ and $0.7 \mathrm{mg}$ ( $P=0.043$ and $P=0.026$ ) in the propofol group (Figure 3 ). Differences in benzodiazepine and haloperidol use between the two groups were not statistically significant $(P=0.933$ and $P=0.465$, respectively).

\section{Secondary measures}

Mean AWAS scores before initiation of sedation were 11.4 $(n=24)$ in the dexmedetomidine group and $12.8(n=2)$ in the propofol group. The AWAS average after initiation of dexmedetomidine was $7.5(\mathrm{n}=18)$. AWAS scores were not available in 15 patients. No patients in the propofol 


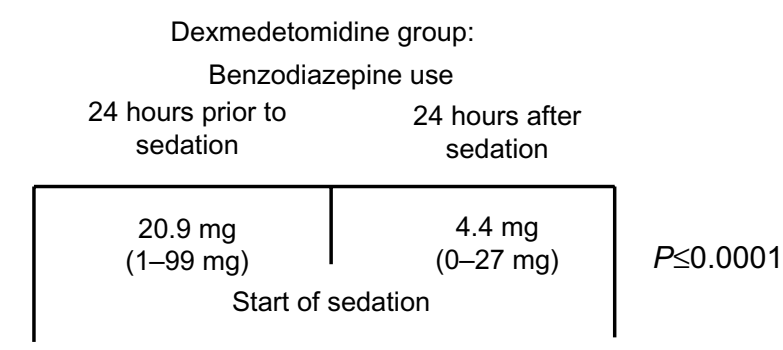
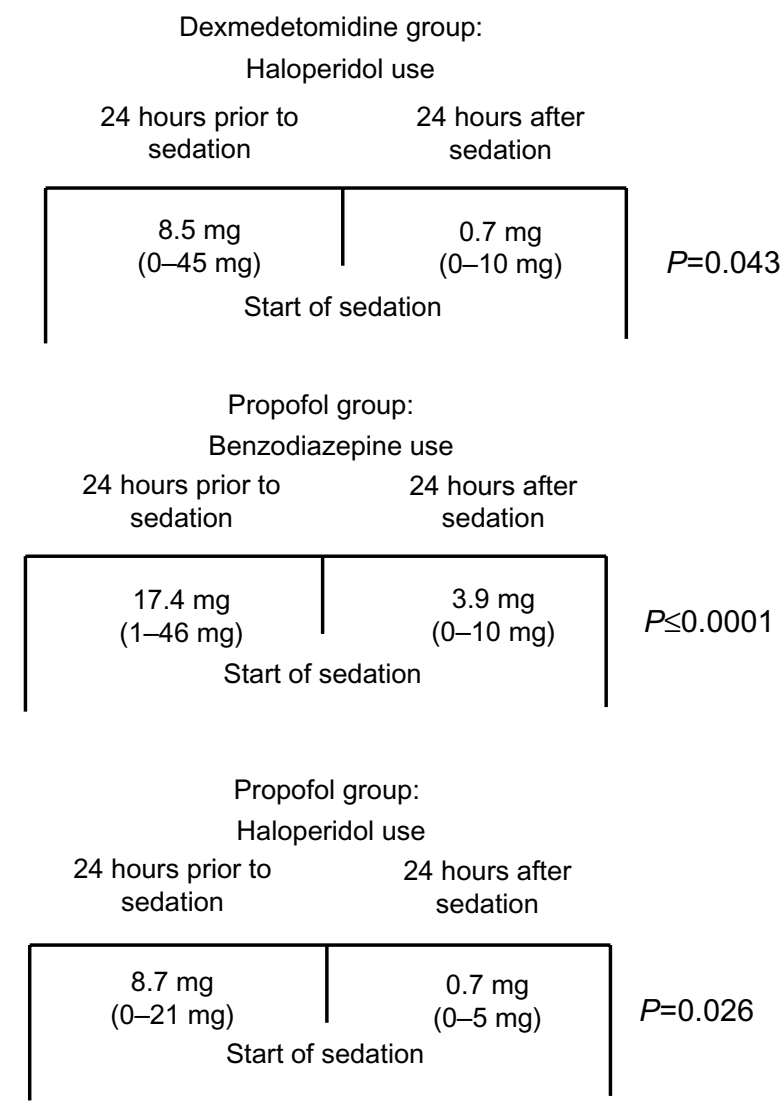

Figure 2 Study timeline and results.

group received follow-up scoring. Average RASS scores were 0.11 and 0.87 in the dexmedetomidine and propofol groups, which decreased to -0.7 and -2.5 , respectively, after initiation of sedation. RASS scores were not available in 12 patients. Of the six patients who had Confusion Assessment Method for the ICU scores performed, all six were positive before initiation of sedation. Only one patient had resolution of delirium after 24 hours of

Table I Equipotent doses of various benzodiazepines

\begin{tabular}{ll}
\hline Benzodiazepine & Equipotent dose \\
\hline Lorazepam - IV/PO & $\mathrm{I} \mathrm{mg}$ \\
Diazepam - IV/PO & $5 \mathrm{mg}$ \\
Chlordiazepoxide - PO & $25 \mathrm{mg}$ \\
\hline
\end{tabular}

Abbreviations: IV, intravenous; PO, orally. dexmedetomidine. There were no differences in analgesic usage between the groups.

\section{Intubation and ICU length of stay}

Five patients (14.7\%) in the dexmedetomidine group and all seven patients $(100 \%)$ in the propofol group were intubated. When intubation was required, intubation times were shorter in the dexmedetomidine group (19.9 hours vs 97.6 hours; $P=0.002$ ). Four of the five intubated patients in the dexmedetomidine group were extubated during the infusion. There was no statistically significant reduction in ICU length of stay between the groups (123.6 hours vs 156.5 hours; $P=0.125$ ).

\section{Adverse events}

Bradycardia and hypotension were the only adverse effects evaluated in the analysis (Table 2). Six patients (17.6\%) in the dexmedetomidine group developed bradycardia and six (17.6\%) developed hypotension. Dexmedetomidine was discontinued due to hypotension in one patient $(2.8 \%)$. Of the six patients who developed hypotension, none of the patients received a bolus dose, and continuous infusion doses ranged from $0.4 \mu \mathrm{g} / \mathrm{kg} / \mathrm{h}$ to $0.8 \mu \mathrm{g} / \mathrm{kg} / \mathrm{h}$. The effects of dexmedetomidine on hypotension were seen immediately upon initiation of the infusion and were typically accompanied by slight improvement within a couple of hours. Among patients who developed bradycardia, a drop in heart rate was seen upon initiation of the infusion, followed by slow progression to bradycardia. Bradycardia did not resolve unless the infusion rate was decreased or the infusion was discontinued. No patients in the propofol group developed bradycardia. Two patients in the propofol group developed hypotension $(28.5 \%)$ during therapy. Hypotension developed in both patients at a dose of $50 \mu \mathrm{g} / \mathrm{kg} / \mathrm{min}$. Blood pressure improved in both patients after reduction of infusion. Propofol was not discontinued in any patient due to adverse events. Three deaths occurred in study patients, one in the dexmedetomidine group and two in the propofol group. All deaths were related to end-stage liver disease and not attributed to the use of either sedative agent.

\section{Discussion}

Both dexmedetomidine and propofol significantly decreased benzodiazepine and haloperidol requirements in patients with AW. The overall reduction in benzodiazepine and haloperidol requirements was not different when comparing dexmedetomidine and propofol. Small sample size limits speculation on the disparity of benzodiazepine dosage range between each group. 

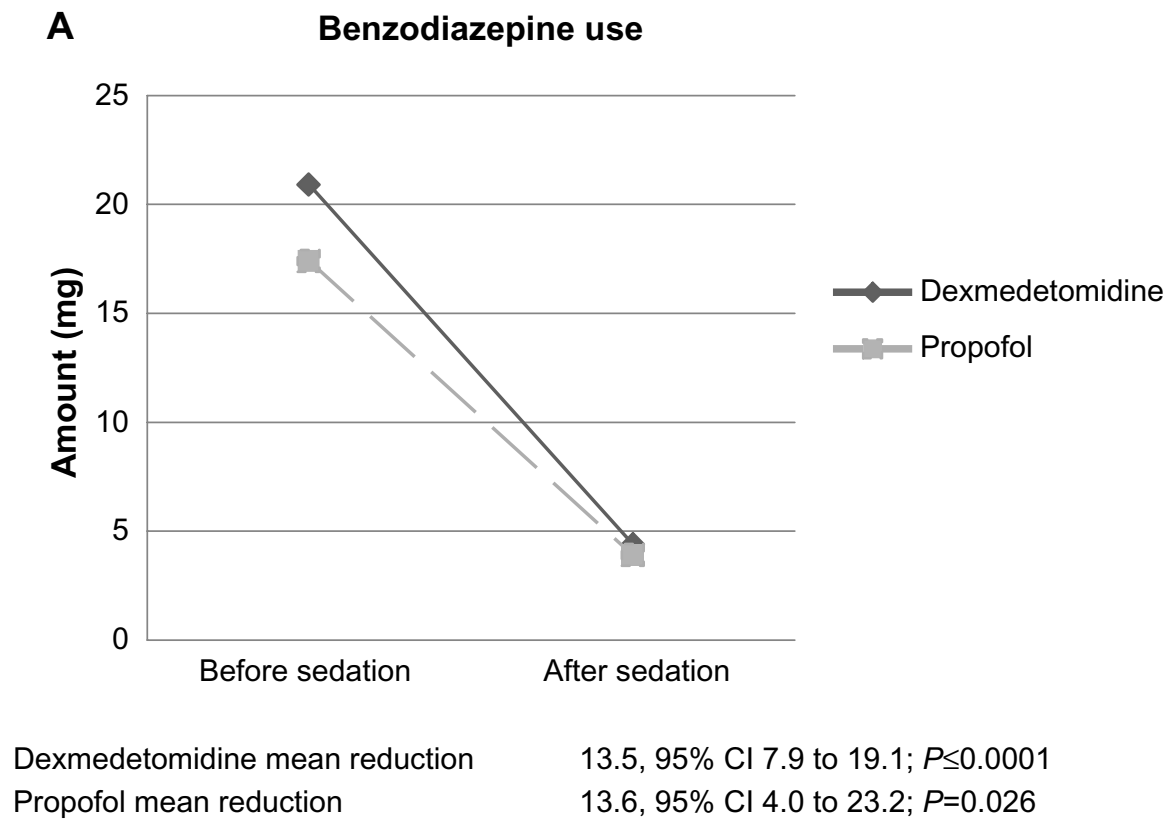

B Haloperidol use

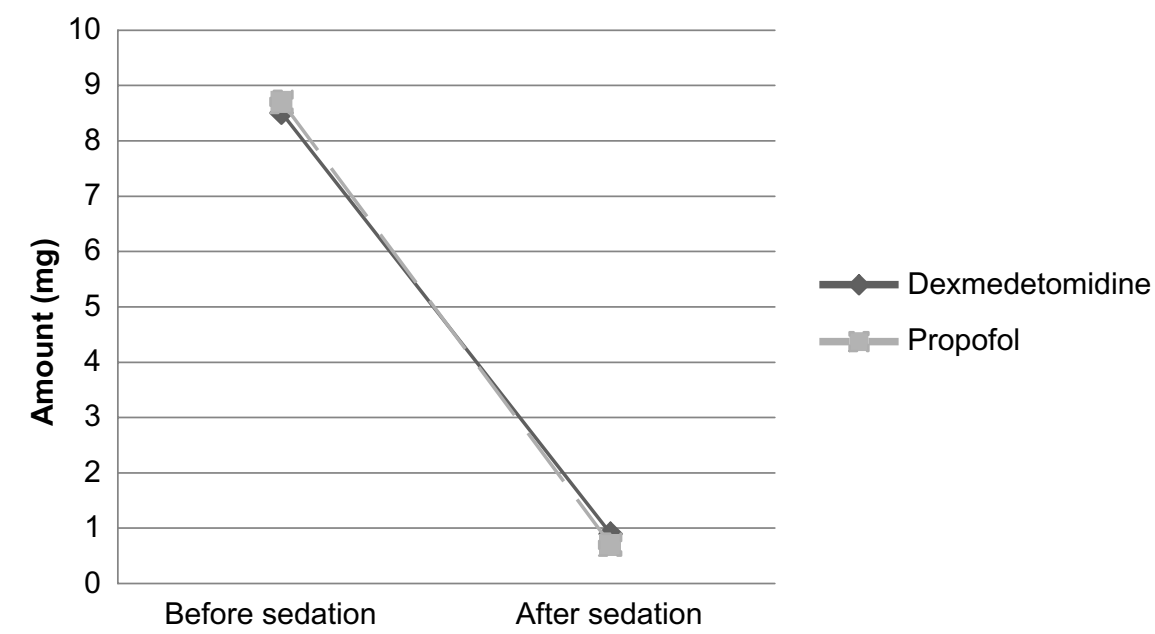

Dexmedetomidine mean reduction

Propofol mean reduction
7.6, $95 \% \mathrm{Cl} 2.0$ to $13.2 ; P \leq 0.0001$

$8.0,95 \% \mathrm{Cl} 4.2$ to $11.8 ; P=0.043$

Figure 3 (A) Benzodiazepine reduction and (B) haloperidol reduction.

Abbreviation: $\mathrm{Cl}$, confidence interval.

However, mean dosing is similar; therefore, meaningful conclusions may still be drawn. Patients in the dexmedetomidine group trended toward shorter ICU length of stay $(P=0.125)$. Only five (14.7\%) patients in the dexmedetomidine group were intubated, with four being extubated while receiving dexmedetomidine. All seven patients in the propofol group were intubated while receiving propofol. When intubated, patients in the dexmedetomidine group were extubated sooner than those in the propofol group $(P=0.002)$. However, it should be noted that type 1 error is possible due to the small number of intubated patients. Patients did experience more bradycardia and hypotension during dexmedetomidine therapy, with one patient requiring discontinuation due to adverse events.

While dexmedetomidine and propofol displayed reductions in benzodiazepine and haloperidol dosing, this may have been mediated through symptom control that reduced AWAS scores, rather than directly alleviating AW symptoms. However, given their therapeutic mechanisms, dexmedetomidine and propofol would provide benefits in patients with AW. Dexmedetomidine acts as a selective $\alpha-2$ adrenergic receptor 
Table 2 Adverse events

\begin{tabular}{llll}
\hline & $\begin{array}{l}\text { Bradycardia } \\
\text { (HR less } \\
\text { than } 60 \mathrm{bpm})\end{array}$ & $\begin{array}{l}\text { Hypotension } \\
\text { (SBP less than } \\
\mathbf{9 0} \mathbf{~ m m H g})\end{array}$ & $\begin{array}{l}\text { Discontinued } \\
\text { due to ADR }\end{array}$ \\
\hline $\begin{array}{l}\text { Dexmedetomidine } \\
(\mathrm{n}=34)\end{array}$ & $6(17.6 \%)$ & $6(17.6 \%)$ & $\mathrm{I}(2.8 \%)$ \\
Propofol $(\mathrm{n}=7)$ & $0(0 \%)$ & $2(28.5 \%)$ & $0(0 \%)$ \\
\hline
\end{tabular}

Abbreviations: HR, heart rate; SBP, systolic blood pressure; ADR, adverse drug reaction; bpm, beats per minute.

agonist, ${ }^{39-42}$ working via a negative feedback mechanism to regulate norepinephrine release. ${ }^{12,24,25,43}$ The locus coeruleus, located in the pons, is responsible for regulating sleep and vigilance and contains a high density of $\alpha-2$ receptors and noradrenergic neurons. ${ }^{12,24}$ These receptors and neurons are responsible for the antinociceptive and sedative effects. ${ }^{44,45}$ In addition, dexmedetomidine alleviates symptoms of AW through inhibition of noradrenergic hyperactivity, including tachycardia, hypertension, and tremulousness. ${ }^{25,43}$ Propofol works similar to alcohol and benzodiazepines as a positive modulator of GABA receptors and inhibition of NMDA receptors. ${ }^{18,46-48}$ These effects may counteract the up- and downregulation of receptors seen in chronic alcohol abuse and prevent AW-related seizures. ${ }^{16-18,49}$

Among the limitations of this study is the retrospective design, which occasionally provided us with incomplete data for secondary objectives. The sample size was small $(n=41)$, which prevents definitive conclusions from being drawn. However, the 34 patients in the dexmedetomidine group is one of the largest populations evaluated for the use of dexmedetomidine in AW to date. The sample of seven patients in the propofol group is also too small to draw conclusions, and also limits evaluation with uneven distribution of patients between groups. The utilization of the AWAS, which incorporates a variety of nonspecific symptoms including anxiety, agitation, and sweating, also serves as a limitation to our results. These symptoms can manifest in a variety of settings, leading to increased scores and dosing for symptoms unrelated to AW. Also, despite standardization in scoring systems, there is still the possibility of subjectivity, especially in a retrospective review. Doses were reviewed for patients who developed adverse events, but the average dose for all patients was not reviewed. Dexmedetomidine doses ranged from $0.4 \mu \mathrm{g} / \mathrm{kg} / \mathrm{h}$ to $1.2 \mu \mathrm{g} / \mathrm{kg} / \mathrm{h}$, and propofol doses ranged from $20 \mu \mathrm{g} / \mathrm{kg} / \mathrm{min}$ to $70 \mu \mathrm{g} / \mathrm{kg} / \mathrm{min}$ with reductions in benzodiazepine and haloperidol dosing after initiation of sedation for both higher and lower infusion rates.

This study is unique in the comparison of dexmedetomidine to propofol in patients with severe AW. To date, the efficacy of dexmedetomidine has only been evaluated in case reports, retrospective reviews with no comparison group, ${ }^{31-36}$ and a single double-blind, placebo-controlled trial. ${ }^{37}$ Propofol for refractory AW has only been evaluated in case reports. ${ }^{18-21,30}$ This study confirms these reports and shows the potential benefits of dexmedetomidine and propofol in AW, although larger, more definitive trials should be performed to verify the results.

\section{Conclusion}

Severe cases of AW may necessitate the addition of a sedative agent to control symptoms. Although our sample size is too small to draw definitive conclusions, this analysis, in addition to previous studies, supports the use of adjunctive dexmedetomidine and propofol in severe AW. Both dexmedetomidine and propofol appear to significantly reduce AW-related symptoms and benzodiazepine and haloperidol requirements. These results should be validated in a larger, prospective trial.

\section{Acknowledgments}

The authors would like to thank Joe Strain, PharmD; Janeen Bucholz, PharmD; Denise Baldwin; Jeremiah Brickhouse; Surachat Ngorsuraches; and Brittany Schaffer for their assistance in performing this study.

\section{Disclosure}

The authors report no conflicts of interest in this work.

\section{References}

1. Mayo-Smith MF, Beecher LH, Fischer TL, et al. Management of alcohol withdrawal delirium: an evidence-based practice guideline. Arch Intern Med. 2004;164(13):1405-1412.

2. McKeon A, Frye MA, Delanty N. The alcohol withdrawal syndrome. J Neurol Neurosurg Psychiatry. 2008;79:854-862.

3. Hall W, Zador D. The alcohol withdrawal syndrome. Lancet. 1997; 349(9069):1897-1900.

4. Ramos R, Mallet T, DiVittis A, Cohen R. Predictors of severity of alcohol withdrawal in hospitalized patients. J Clin Med Res. 2013;5(5): 376-380.

5. Treiman DM. GABAergic mechanisms in epilepsy. Epilepsia. 2001;42(Suppl 3):8-12.

6. Richards G, Schoch P, Haefely W. Benzodiazepine receptors: new vistas. Semin Neurosci. 1991;3:191-203.

7. Hack JB, Hoffman RS, Neslon LS. Resistant alcohol withdrawal: does an unexpectedly large sedative requirement identify these patients early? J Med Toxicol. 2006;2(2):55-60.

8. Nolop KB, Natow A. Unprecedented sedative requirements during delirium tremens. Crit Care Med. 1985;13(4):246-247.

9. Lineaweaver WC, Anderson K, Hing DN. Massive doses of midazolam infusion for delirium tremens without respiratory depression. Crit Care Med. 1988;16(3):294-295.

10. Kahn DR, Barnhorst AV, Bourgeois JA. A case of alcohol withdrawal requiring 1,600 $\mathrm{mg}$ of lorazepam in 24 hours. CNS Spectr. 2009;14(7): 385-389.

11. Pandharipande P, Shintani A, Peterson J, et al. Lorazepam is an independent risk factor for transitioning to delirium in intensive care unit patients. Anesthesiology. 2006;104(1):21-26. 
12. Pertuzzi WT. Sleep in the intensive care unit. Pharmacotherapy. 2005;25(5 pt 2):34S-39S.

13. Gommers D, Bakker J. Medications for analgesia and sedation in the intensive care unit: an overview. Crit Care. 2008;12(Suppl 3):S4.

14. Hall RI, Sandham D, Cardinal P, et al. Propofol vs midazolam for ICU sedation: a Canadian multicenter randomized trial. Chest. 2001;119: 1151-1159.

15. Ostermann ME, Keenan SP, Seiferling RA, Sibbald WJ. Sedation in the intensive care unit: a systematic review. JAMA. 2000;283(11): 1451-1459.

16. Kofke WA. Anesthetic management of the patient with epilepsy or prior seizures. Curr Opin Anaesthesiol. 2010;23(3):391-399.

17. MacKenzie S, Kapadia F, Grant IS. Propofol infusion for control of status epilepticus. Anaesthesia. 1990;45(12):1043-1045.

18. McCowan C, Marik P. Refractory delirium tremens treated with propofol: a case series. Crit Care Med. 2000;28(6):1781-1784.

19. Coomes TR, Smith SW. Successful use of propofol in refractory delirium tremens. Ann Emerg Med. 1997;30(6):825-828.

20. Takeshita J. Use of propofol for alcohol withdrawal delirium: a case report. J Clin Psychiatry. 2004;65(1):134-135.

21. Sohraby R, Attridge RL, Hughes DW. Use of propofol-containing versus benzodiazepine regimens for alcohol withdrawal requiring mechanical ventilation. Ann Pharmacother. 2014;48(4):456-461.

22. Precedex. [package insert]. Lake Forest, IL: Hospira, Inc.; 2012.

23. Gerlach AT, Murphy CV, Dasta JF. An updated focused review of dexmedetomidine in adults. Ann Pharmacother. 2009;43(12):2064-2074.

24. Gertler R, Brown C, Mitchell DH, Silvius EN. Dexmedetomidine: a novel sedative-analgesic agent. Proc (Bayl Univ Med Cent). 2001;14:13-21.

25. Muzyk AJ, Fowler JA, Norwood DK, Chilipko A. Role of alpha2-agonists in the treatment of acute alcohol withdrawal. Ann Pharmacother. 2011;45(5):649-657.

26. Ebert TJ, Hall JE, Barney JA, Uhrich TD, Colinco MD. The effects of increasing plasma concentrations of dexmedetomidine in humans. Anesthesiology. 2000;93(2):382-394.

27. Jakob SM, Ruokonen E, Grounds RM, et al. Dexmedetomidine vs midazolam or propofol for sedation during prolonged mechanical ventilation. JAMA. 2012;307(11):1151-1160.

28. Pandharipande PP, Pun BT, Herr DL, et al. Effect of sedation with dexmedetomidine vs lorazepam on acute brain dysfunction in mechanically ventilated patients: the MENDS randomized controlled trial. JAMA. 2007;298(22):2644-2653.

29. Siobal MS, Kallet RH, Kivett RH, Tang JF. Use of dexmedetomidine to facilitate extubation in surgical intensive-care-unit patients who failed previous weaning attempts following prolonged mechanical ventilation: a pilot study. Respir Care. 2006;51(5):492-496.

30. Hughes DW, Vanwert E, Lepori L, Adams BD. Propofol for benzodiazepine-refractory alcohol withdrawal in a non-mechanically ventilated patient. Am J Emerg Med. 2014;32(1):112.e3-112.e4.

31. Rayner SG, Weinert CR, Peng H, Jepson S, Broccard AF, Study institution. Dexmedetomidine as adjunct treatment for severe alcohol withdrawal in the ICU. Ann Intensive Care. 2012;2(1):12.

32. Darrouj J, Puri N, Prince E, Lomonaco A, Spevetz A, Gerber DR. Dexmedetomidine infusion as adjunctive therapy to benzodiazepines for acute alcohol withdrawal. Ann Pharmacother. 2008;42(11): 1703-1705.
33. Demuro JP, Botros DG, Wirkowski E, Hanna AF. Use of dexmedetomidine for the treatment of alcohol withdrawal syndrome in critically ill patients: a retrospective case series. J Anesth. 2012;26(4): 601-605.

34. Frazee EN, Personett HA, Leung JG, Nelson S, Dierkhising RA, Bauer PR. Influences of dexmedetomidine therapy on the management of severe alcohol withdrawal syndrome in critically ill patients. J Crit Care. 2014;29(2):298-302.

35. Rovasalo A, Tohmo H, Aantaa R, Kettunen E, Palojoki R. Dexmedetomidine as an adjunct in the treatment of alcohol withdrawal delirium: a case report. Gen Hosp Psychiatry. 2006;28(4):362-363.

36. Crispo AL, Daley MJ, Pepin JL, Harford PH, Brown CV. Comparison of clinical outcomes in non intubated patients with severe alcohol withdrawal syndrome treated with continuous-infusion sedatives: dexmedetomidine versus benzodiazepines. Pharmacotherapy. 2014;34(9):910-917.

37. Mueller SW, Preslaski CR, Kiser TH, et al. A randomized, doubleblind, placebo-controlled dose range study of dexmedetomidine as adjunctive therapy for alcohol withdrawal. Crit Care Med. 2014;42(5): 1131-1139.

38. Kane SP. Benzodiazepine equivalents conversion calculator. ClinCalc. [Updated February 11, 2014]. Available from: http://clincalc.com/ Benzodiazepine/. Accessed April 23, 2014.

39. Savola JM, Virtanen R. Central alpha 2-adrenoreceptors are highly stereoselective for dexmedetomidine, the dextro enantiomer of medomidine. Eur J Pharmacol. 1991;195(2):193-199.

40. Furst SR, Weinger MB. Dexmedetomidine, a selective alpha 2-agonist, does not potentiate the cardiorespiratory depression of alfentanil in the rat. Anesthesiology. 1990;72(5):882-888.

41. Tobias JD. Dexmedetomidine: are there going to be issues with prolonged administration? J Pediatr Pharmacol Ther. 2010;15(1):4-9.

42. Bischoff P, Kochs E. Alpha 2-agonists in anesthesia and intensive medicine. Anasthesiol Intensivmed Nofallmed Schmerzther. 1993;28(1): $2-12$.

43. Jaatinen P, Riihioja P, Haapalinna A, Heinonen E, Kiianmaa K, Hervonen A. Prevention of ethanol-induced sympathetic overactivity and degeneration be dexmedetomidine. Alcohol. 1995;12(5):439-446.

44. Hunter JC, Fontana DJ, Hedley LR, et al. Assessment of the role of alpha2-adrenoceptor subtypes in the antinociceptive, sedative, and hypothermic action of dexmedetomidine in transgenic mice. $\mathrm{Br} J$ Pharmacol. 1997;122(7):1339-1344.

45. Correa-Sales C, Rabin BC, Maze M. A hypnotic response to dexmedetomidine, an alpha 2 agonists, is mediated in the locus coeruleus in rats. Anesthesiology. 1992;76(6):948-952.

46. Orser BA, Bertlik M, Wang LY, MacDonald JF. Inhibition by propofol (2,6 di-isopropylphenol) of the $N$-methyl D-aspartate subtype of glutamate receptor in cultured hippocampal neurons. Br J Pharmacol. 1995;116(2):1761-1768.

47. Uchida I, Li L, Yang J. The role of the GABA(A) receptor alpha1 subunit N-terminal extracellular domain in propofol potentiation of chloride current. Neuropharmacology. 1997;36:1611-1621.

48. Hara M, Kai Y, Ikemoto Y. Propofol activates GABA-A receptorchloride ionophore complex in dissociated hippocampal pyramidal neurons of the rat. Anesthesiology. 1993;79:781-788.

49. Marik PE, Varon J. The management of status epilepticus. Chest. 2004;126(2):582-591.

Clinical Pharmacology: Advances and Applications

\section{Publish your work in this journal}

Clinical Pharmacology: Advances and Applications is an international, peer-reviewed, open access journal publishing original research, reports, reviews and commentaries on all areas of drug experience in humans. The manuscript management system is completely online and includes a very quick and fair peer-review system, which is all easy to use.

Dovepress

Visit http://www.dovepress.com/testimonials.php to read real quotes from published authors. 